\title{
Alternatives Verfahren zur Tumorkontrolle
}

Zur lokalen Kontrolle inoperabler oder multipler Hauttumoren nutzt die Hautklinik der Universitätsmedizin Mainz seit kurzem regelhaft die so genannte Elektrochemotherapie (ECT). Die Vorgehensweise beinhaltet die intravenöse oder intratumorale Injektion eines Chemotherapeutikums, gefolgt von einem kurzen Stromimpuls von hoher Intensität. Dadurch gelangt die Substanz in bis zu 5000-facher Konzentration in die Tumorzelle. Auf diese Weise lässt sich potentiell eine weitere Tumorausbreitung verhindern oder die Tumormasse verkleinern.

Die lokale Tumorkontrolle ist wichtig, um zu verhindern, dass sich vom primären Entstehungsort eines Tumors aus Metastasen bilden. Dies ermöglicht die so genannte Elektrochemotherapie (ECT) für als inoperabel geltende Hauttumoren, metastasierende maligne Melanome der Haut, metastasierende Mammakarzinome oder für multiple Tumoren der Haut. Der Eingriff geschieht meist in Vollnarkose oder unter lokaler Betäubung bei kleineren Befunden.

\section{Zellmembran wird durchlässiger}

Bei der ECT wird ein Chemotherapeutikum verabreicht. In der Regel handelt es sich dabei um Bleomycin oder Cisplatin, welches entweder intravenös oder intratumoral gegeben werden kann. Die Gabe dieser Arzneistoffe ist von einem kurzen, aber sehr intensiven Stromstoß begleitet. Durch diesen können die Chemotherapeutika in bis zu 5000-facher Konzentration in die Tumorzellen hineinströmen. Denn der Stromimpuls bewirkt, dass die Zellmembran vorübergehend für die Arzneistoffe durchlässig ist.

》) Der Eingriff geschieht meist in Vollnarkose oder unter lokaler Betäubung

Quelle: Pressemitteilung der Unimedizin Mainz, Firma IGEA
Tumorzellen verbleibt und umfassender wirkt. „Durch die Hydrophilie beider Substanzen - Bleomycin oder Cisplatin - kommt es zu Interaktionen mit der Tumor-DNA, die den Zelltod herbeiführen", so Dr. Hadrian Schepler von der Hautklinik und Poliklinik der Universitätsmedizin Mainz, der das Verfahren schon mehrfach erfolgreich angewandt hat.

\section{Alternative zu bisherigen lokalen Eingriffen}

Die ECT stellt eine Alternative zu anderen lokalen Verfahren wie der Radiotherapie oder der Operation dar, sofern sich durch diese keine ausreichende Tumorkontrolle erzielen lässt. Darüber hinaus kommt das Verfahren zum Einsatz, wenn eine bereits vorbestrahlte Region nicht mehr anderweitig behandelbar ist oder die Lebensqualität stark beinflussende Operationen nicht vertretbar sind.

\) Offene und schmierig belegte Tumore lassen sich mit dieser Methode gut eingrenzen

„Da es sich um einen rein lokalen Eingriff handelt, bleiben ergänzende oder unterstützende systemische Therapiemaßnahmen davon unberührt", so Schepler. „Die Verkleinerung großer oder multilokaler Tumormassen unterstützt neuesten Studien zufolge sogar die Wirkung von sogenannten Sys- temtherapien gegen Krebs." Auch lassen sich mithilfe der ECT Tumorblutungen stoppen beziehungsweise abmildern. Offene, oft unangenehm riechende und schmierig belegte Tumore lassen sich mit dieser Methode gut eingrenzen. „Gerade in palliativen Situationen bedeutet das eine erhebliche Steigerung der Lebensqualität", bemerkt Schepler.

\section{Nebenwirkungen}

Die Nebenwirkungen dieses Verfahrens beschränken sich meist auf anschließende muskelkaterartige Beschwerden, Übelkeit nach der Gabe von Cisplatin oder einem leichten Brennen an den behandelten Stellen. Schwerwiegende Nebenwirkungen sind nicht bekannt.

Insgesamt sind leichte Nebenwirkungen mit Rötungen und lokalen Schmerzen auch bei älteren, multimorbiden Patienten mit drei bis vier Prozent als niedrig zu verzeichnen. Die Ergebnisse nach ECT sind vielversprechend. Abheilungsraten von über $80 \%$ sind veröffentlicht.

hautnah $2019 \cdot 18: 22$

https://doi.org/10.1007/s12326019-0321-0

(c) Springer-Verlag GmbH Austria, ein Teil von Springer Nature 2019 